OPEN ACCESS

Edited by:

Andrea Tipold,

University of Veterinary Medicine

Hanover, Germany

Reviewed by:

Christopher R. Lamb,

Royal Veterinary College (RVC), UK

Elsa Beltran,

Royal Veterinary College (RVC), UK

${ }^{*}$ Correspondence:

Krystina L. Stadler

krystina@vt.edu

Presented in abstract form at the American College of Veterinary Radiology Annual Conference, Minneapolis, MN, USA, 2015.

tPresent address:

Krystina L. Stadler,

Department of Small Animal Clinical Sciences, Virginia-Maryland College of Veterinary Medicine, Blacksburg, $V A$, USA

Specialty section: This article was submitted to Veterinary Imaging,

a section of the journal

Frontiers in Veterinary Science

Received: 23 November 2016 Accepted: 07 March 2017

Published: 21 March 2017

Citation:

Stadler KL, Pease AP and Ballegeer EA (2017) Dynamic

Susceptibility Contrast Magnetic Resonance Imaging Protocol of the Normal Canine Brain. Front. Vet. Sci. 4:41. doi: 10.3389/fvets.2017.00041

\section{Dynamic Susceptibility Contrast Magnetic Resonance Imaging Protocol of the Normal Canine Brain}

\author{
Krystina L. Stadler ${ }^{\star 1}$, Anthony P. Pease and Elizabeth A. Ballegeer
}

Department of Small Animal Clinical Sciences, Michigan State University College of Veterinary Medicine, East Lansing, MI, USA

Perfusion magnetic resonance imaging (MRI), specifically dynamic susceptibility MRI (DSC-MRI) is routinely performed as a supplement to conventional MRI in human medicine for patients with intracranial neoplasia and cerebrovascular events. There is minimal data on the use of DSC-MRI in veterinary patients and a DSC-MRI protocol in the veterinary patient has not been described. Sixteen normal dogs, 6 years or older were recruited for this study. The sample population included 11 large dogs (>11 kg) and 5 small dogs $(<11 \mathrm{~kg})$. DSC-MRI was performed on a 1.5-T MRI using an adjusted protocol inherent to the MRI. Contrast media was injected using an automatic power injector. Injections were made after five MR measurements were obtained. Following image acquisition, an arterial input function (AIF) graph mapping the transit time of contrast within the cerebral arteries was generated. The manually selected time points along this graph were used to compute perfusion maps. A dose and rate of $0.1 \mathrm{mmol} / \mathrm{kg}$ gadolinium-based contrast media at $3 \mathrm{ml} / \mathrm{s}$ followed by $10 \mathrm{ml}$ saline flush at $3 \mathrm{ml} / \mathrm{s}$ was used in all dogs greater than $11 \mathrm{~kg}$. In all dogs $>11 \mathrm{~kg}$, a useable AIF and perfusion map was generated. One dog less than $11 \mathrm{~kg}$ received the same contrast dose and rate. In this patient, the protocol did not generate a useable AIF. The remainder of the dogs less than $11 \mathrm{~kg}$ followed a protocol of $0.2 \mathrm{mmol} / \mathrm{kg}$ gadolinium-based contrast media at $1.5 \mathrm{ml} / \mathrm{s}$ with a $10 \mathrm{ml}$ saline flush at $1.5 \mathrm{ml} / \mathrm{s}$. A useable AlF and perfusion map was generated in the remaining dogs $<11 \mathrm{~kg}$ using the higher contrast dose and slower rate protocol. This study establishes a contrast dose and administration rate for canine DSC-MRI imaging that is different in dogs greater than $11 \mathrm{~kg}$ compared to dogs less than $11 \mathrm{~kg}$. These protocols may be used for future applications to evaluate hemodynamic disturbances in canine intracranial pathology.

Keywords: canine, perfusion, dynamic susceptibility contrast, magnetic resonance imaging, neuroimaging

Abbreviations: AIF, arterial input function; CBF, cerebral blood flow; CBV, cerebral blood volume; DCE-MRI, dynamic contrast-enhanced magnetic resonance imaging; DSC-MRI, dynamic susceptibility contrast magnetic resonance imaging; FLAIR, fluid attenuating inversion recovery; MRI, magnetic resonance imaging; MTT, mean transit time; ROI, region of interest; T1W, T1-weighted; T2 $\left({ }^{*}\right) \mathrm{W}, \mathrm{T} 2\left(^{*}\right)$-weighted. 


\section{INTRODUCTION}

Perfusion magnetic resonance imaging (MRI) is an important non-invasive tool in human medicine for evaluating cerebral hemodynamics (1). Two contrast-based perfusion imaging sequences are described: dynamic susceptibility weighted dynamic susceptibility MRI (DSC-MRI) and dynamic contrastenhanced MRI (DCE-MRI) (2). In human medicine, DSC-MRI is the most widely used method to measure brain perfusion due to the software availability and ease of use $(2,3)$.

Dynamic susceptibility MRI images the first pass of a bolus of gadolinium-based contrast through the brain by a series of $\mathrm{T} 2{ }^{\star}$-weighted $\left(\mathrm{T} 2{ }^{\star} \mathrm{W}\right) \mathrm{MRI}$ images to generate a signal intensity to time curve, also known as an arterial input function (AIF). The susceptibility of the contrast causes a decrease in signal intensity, which leads to a signal loss in the AIF. From this curve, multiple hemodynamic parameters such as time to peak, mean transit time (MTT), cerebral blood flow (CBF), and cerebral blood volume (CBV) can be determined for each pixel and perfusion maps are generated $(2,4)$. A major assumption in DSC-MRI studies is that contrast remains within the blood vessels such that a susceptibility gradient can be induced between the intravascular and extravascular space, this assumption can lead to underestimation of perfusion, specifically in brain tumors $(5,6)$. DCE-MRI allows for better quantitative measurement of the blood-brain barrier, assessing the tissue permeability and the extracellular space, the values are sensitive to tumor growth and treatment response (7). DCE-MRI techniques involve serial T1-weighted (T1W) images before, during, and after gadolinium contrast administration (7). The reason DSC-MRI is used more often in the clinical setting is due to the complexity in image acquisition and post-processing of DCE-MRI data and the lack of widely available software (2). In contrast, most commercially available MRI scanners have inherent acquisition parameters and software for DSC-MRI. To the authors' knowledge, the only descriptive study in veterinary medicine using perfusion MRI is a quantitative perfusion study using DCE-MRI in dogs with intracranial neoplasia using 3-T MRI and manual contrast injection (8). Since both DSC-MRI and DCE-MRI involve dynamic imaging acquisitions, the use of an automated power injector is considered necessary to allow a fast injection needed for DSC-MRI and reproducible administration DCE-MRI perfusion (2). No studies using DSC-MRI in the normal canine brain have been described.

Extensive research and clinical applications of DSC-MRI are described in human patients with stroke $(9,10)$, neoplasia (11-13), dementia (14), anesthesia (15), epilepsy (16), and trauma $(17,18)$. Of the described clinical applications of DSC-MRI in human medicine, the use of DSC-MRI in intracranial neoplasia grading and therapeutic monitoring is the most clinically relevant to our canine patients and translational research $(12,19-24)$. Despite the amount of data available in human medicine, few reports in veterinary medicine have been published, with most available studies using animals as a model of disease. Examples include canine studies of ischemic stroke (25) and brain changes secondary to cardiac arrest (26). Within the veterinary literature, to the authors' knowledge, in addition to the previously mentioned DCE-MRI study, two descriptive reviews are available for vascular and perfusion imaging in the canine brain, with no protocol details $(27,28)$.

A protocol for DSC-MRI at $1.5 \mathrm{~T}$ in the normal canine brain with a power injector has not been described. The aim of this study was to determine a DSC-MRI protocol for the normal canine brain.

\section{METHODS}

The study protocol was designed in accordance with and approved by the Michigan State University animal care and use committee. The study was a prospective cohort study performed by recruiting client owned, consented healthy dogs, middle to senior in age ( $\geq 6$ years).

All study dogs underwent a physical exam and bloodwork (complete blood count and serum chemistry) to ensure the animals were healthy and able to undergo anesthesia. All dogs were imaged under general anesthesia. All dogs received butorphanol $(0.2 \mathrm{mg} / \mathrm{kg}, \mathrm{IV}$, or IM) as a premedication prior to induction. Varying by case, acepromazine $(0.05 \mathrm{mg} / \mathrm{kg}$, IV, or IM) was also administered as a premedication. General anesthesia was induced with propofol ( $4 \mathrm{mg} / \mathrm{kg}$, IV, titrated to effect). The dogs were intubated and maintained on light anesthetic plane using sevofluorane gas anesthesia. Depending on patient size, an 18- or 20 -gauge catheter was placed within the cephalic vein approximately $20 \mathrm{~min}$ after premedication administration and prior to anesthetic induction for anesthetic intravenous fluid delivery and contrast bolus administration.

Images were acquired using a 1.5-T Siemens Espree (Melvin, PA, USA) and an 8-channel coil head or knee coil. In general, large dogs were placed in the head coil due to its greater internal diameter and smaller dogs in the knee coil to minimize air gap between the coil and patient. All dogs had an abbreviated pre-contrast conventional brain MRI study including transverse $\mathrm{T} 1 \mathrm{~W}$ and fluid attenuating inversion recovery sequences.

Dynamic susceptibility MRI images were acquired using a first pass gadolinium contrast-enhanced $\mathrm{T} 2{ }^{*} \mathrm{~W}$ echo-planar image sequence (Siemens, ep2d_perf) with 50 measurements. Each measurement ranged from 1.5 to $2 \mathrm{~s}$ long depending on number of slices. Continuous transverse slices throughout the brain were made with each measurement. The number of slices was dependent on patient size. The image acquisition parameters were as follows repetition time: average $2,008 \mathrm{~ms}$ (range: 1,690-2,250), echo time: $62.4 \mathrm{~ms}$, flip angle: $90^{\circ}$, slice thickness: $4 \mathrm{~mm}$, field of view: 140 or 150 , number of excitations: 1 , and matrix size of $64 \times 64 \times 16$. Paramagnetic contrast media, gadobenate dimeglumine (Multihance ${ }^{\circledR}$ ), was injected into the cephalic vein catheter using an automatic power injector (Spectris Solaris ${ }^{\circledR}$, Medrad) after five measurements. All dogs greater than $11 \mathrm{~kg}$ received a $0.1 \mathrm{mmol} / \mathrm{kg}$ contrast bolus at $3 \mathrm{ml} / \mathrm{s}$ followed by $10 \mathrm{ml}$ saline flush at $3 \mathrm{ml} / \mathrm{s}$. One dog less than $11 \mathrm{~kg}$ received the same dose and rate. The remainder of dogs less than $11 \mathrm{~kg}$ followed a modified protocol of $0.2 \mathrm{mmol} /$ $\mathrm{kg}$ contrast at $1.5 \mathrm{ml} / \mathrm{s}$ with a $10 \mathrm{ml}$ saline flush at $1.5 \mathrm{ml} / \mathrm{s}$. After perfusion data was obtained, all dogs underwent a post-contrast transverse T1W study. 


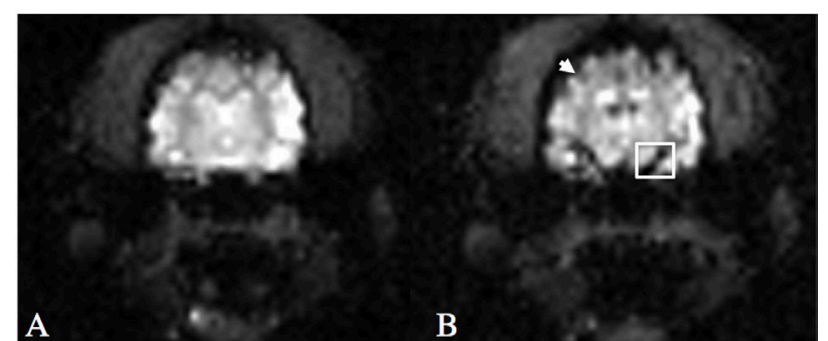

FIGURE 1 | T2*W echo-planar imaging sequence following gadolinium-based contrast injection at $\mathrm{T}=\mathbf{0}(\mathrm{A})$ and time of peak arterial contrast susceptibility $(B)$ at the level of the middle cerebral artery. Note the hypointense cortical arteries around the periphery of the cerebrum (arrow) and the middle cerebral artery (box) at peak contrast susceptibility.
Post-processing of DSC-MRI images was performed using the Siemens MRI analysis software (syngo.MR.NeuroPerfusion ${ }^{\circledR}$ ) by one of the authors (Krystina L. Stadler). This commercially available software generates multiple AIF graphs based on a defined region of interest (ROI) centered over an area of high perfusion. For this study, the middle cerebral artery was used as the ROI (Figure 1). The AIF graphs T2* signal strength ( $y$-axis) against time ( $x$-axis). As the bolus arrives to the ROI, there is a drop-in signal strength (susceptibility on $\mathrm{T} 2{ }^{\star} \mathrm{W}$ images), which remains until redistribution occurs (Figure 2). For further analysis, three of the AIF graphs were selected (Figure 2A). The selection of the three best AIF was subjective, defined by the AIF with the greatest defined peak and least amount of background noise. The graphs are then averaged by the software and used to generative a representative averaged AIF that is used to create cerebral perfusion maps (Figure 2B). From the generated average, AIF, the baseline
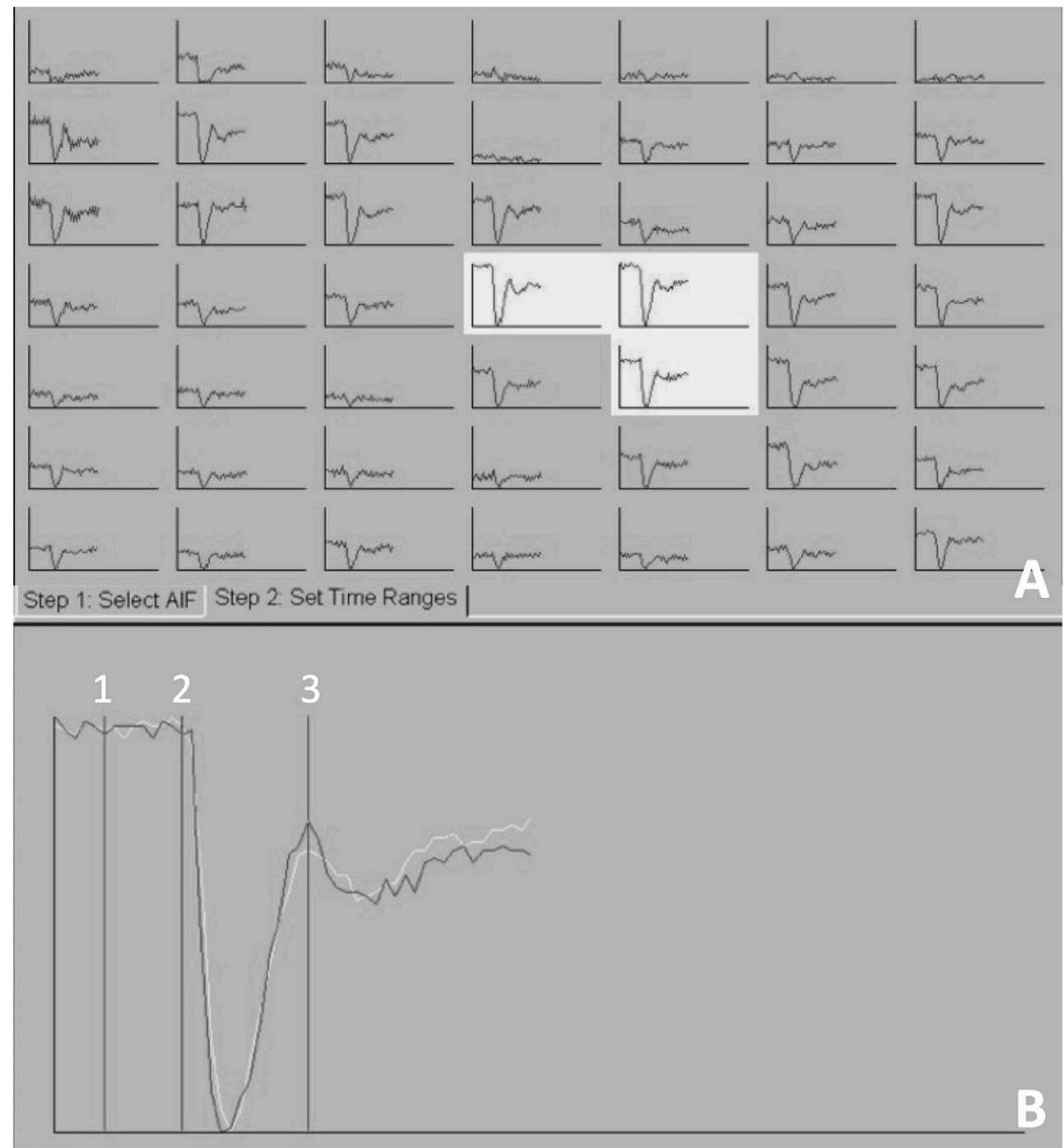

FIGURE 2 | The arterial input function (AIF) generated at the level of the middle cerebral artery, mapping T2*signal ( $y$-axis) against time ( $x$-axis). Three AIF graphs were selected $(\mathbf{A})$ and a representative averaged AIF was generated $(\mathbf{B})$. Three points on the averaged AIF were selected $1-$ the baseline prior to the contrast arrival, 2-point on the graph immediately prior to signal loss peak, and 3-time immediately after return to baseline. 


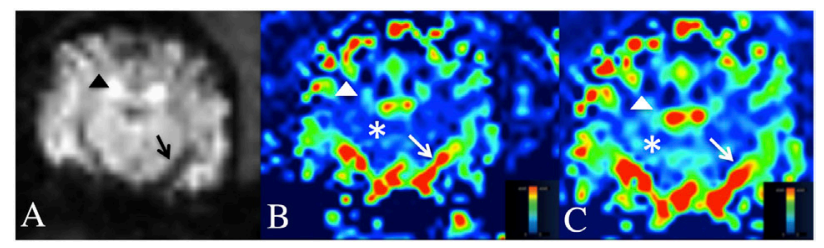

FIGURE 3 | Dynamic susceptibility MRI (DSC-MRI) in the normal canine brain. (A) DSC-MRI T2*W image at the level of the middle cerebral artery. Note the hypointensity of the cortical arteries (black arrow head) and middle cerebral artery (black arrow) during the arterial first pass of the gadolinium contrast bolus. (B,C) Cerebral blood volume (B) and cerebral blood flow (C) maps at the level of the middle cerebral artery. A blue-red scale is used on these maps, where red is high perfusion and blue is low perfusion. Note the red middle cerebral arteries and red-green cortical arteries (white arrow and arrow head) and the blue-green cerebral parenchyma (white asterisks).

prior to the contrast arrival, point on the graph immediately prior to signal loss peak, and time immediately after return to baseline were selected (Figure 2B). After these points are selected, the software generates relative color perfusion maps of the entire brain based on the perfusion imaging physics in which $\mathrm{CBV}$ is represented by the integrated area under the AIF curve, relative MTT is the width of the curve and CBF as the ratio between CBV and MTT. The software uses a blue-green-red color scheme for $\mathrm{CBV}$ and $\mathrm{CBF}$ maps with red being highly perfused, dark blue indicating extremely low perfusion, and green being intermediate perfusion (Figure 3).

\section{RESULTS}

Sixteen dogs met the inclusion criteria for this study. Multiple breeds were represented including: Staffordshire terrier ( $n=4)$, mixed breed dogs (3), Laborador retriever (1 pure, one crossbreed), and one of each the following: Papillion, Beagle, Shiba Inu, Jack Russel Terrier, German Shepherd and Chihuahua. Nine dogs were spayed females, five were neutered males, and one was an intact male. The mean dog weight was $21.2 \mathrm{~kg}$ (range: $3.7-40.4 \mathrm{~kg}$ ). Body condition score was not evaluated.

The injection protocol of $0.1 \mathrm{mmol} / \mathrm{kg}$ gadolinium followed by $10 \mathrm{ml}$ saline flush at an injection rate of $3 \mathrm{ml} / \mathrm{s}$ generated a useable perfusion map in all dogs $>11 \mathrm{~kg}(n=11)$. In a small dog, weighing $10.5 \mathrm{~kg}$, this protocol did not generate a useable map. This dog had evidence of contrast administration noted on the post-contrast T1W images. Following this dog, the protocol was augmented and a useable perfusion map was generated in the remaining dogs weighing less than $11 \mathrm{~kg}(n=4)$ receiving the $0.2 \mathrm{mmol} / \mathrm{kg}$ of contrast medium and $10 \mathrm{ml}$ saline flush at an injection rate of $1.5 \mathrm{ml} / \mathrm{s}$ protocol.

On the CBV and CBF maps, the large arterial structures (cerebral and cortical arteries) of the cerebrum were red on perfusion maps (high perfusion) with the smaller peripheral branches of these structures being green. The normal cerebral parenchyma was predominately royal blue (low perfusion) with small foci of light blue (low, but slightly higher perfusion).
Areas of no perfusion, such as the lateral ventricles were dark blue.

\section{DISCUSSION}

The main clinical indications for perfusion imaging are neoplasia or a cerebrovascular event. Therefore, dogs 6 years or older were selected for this study to best match the age of dogs more commonly affected with intracranial diseases diagnosed by DSCMRI. In human medicine, quantitative MRI CBF changes with age; however, in adults, qualitative maps are generated without changing protocol (29). This study did not validate this protocol in dogs younger than 6 years. The cause of the $0.1 \mathrm{mmol} / \mathrm{kg}$ at $3 \mathrm{ml} / \mathrm{s}$ protocol failure in one of the small dogs is unknown. This dog was the first small dog to be included in the study and was imaged after multiple successful DSC-MRI in dogs larger than this one. The augmentation of the protocol for smaller dogs was based on protocols successfully used in neonatal humans and piglets $(1,30,31)$. Ideally, additional small dogs would have been tested at the protocol to see if the failure occurred repeatedly, however, given the funding available and limited availability of small dogs fitting our inclusion criteria, this was not possible and is a limitation to this study. The dog that failed to generate perfusion maps was $10.5 \mathrm{~kg}$ and thus the threshold weight limit between small and large dogs was placed at $11 \mathrm{~kg}$. The closest large dog weight to the $11 \mathrm{~kg}$ cutoff was $15 \mathrm{~kg}$; this dog generated a useable AIF with the lower contrast dose, higher rate protocol. The authors postulate that the small volume of contrast and/or the rate of contrast administration are a contributing factor of the lower contrast/higher rate DSC-MRI protocol not working in the small dogs. In the authors' clinical experience, the lower contrast dose/higher rate was not successful in additional small dogs; these dogs were not included in this study because they had a known intracranial disease, which may have resultant alterations in their cerebrovascular perfusion. Dosing by $\mathrm{mmol} /$ body weight $(\mathrm{kg})$ is convention at our institution. Body condition score was not factored into contrast dosing. To the author's knowledge, the relationship between patient body condition and gadolinium-based contrast media dosage has not been described. It is unknown if altering the DSC-MRI protocol for ideal body condition weight in patients that are over or under conditioned into the small or large dog protocol would affect the AIF and is a limitation of this study.

In this study, the middle cerebral artery was chosen to generate the AIF because of its reproducible susceptibility that occurred on arterial first pass and for continuity in map generation. Although not assessed in this study, the placement of the ROI on any large artery, preferably in the same slice as your pathology is recommended in human medicine and should generate a similar perfusion map (32). This study's aim was to describe a contrast protocol that can be used in the canine patients for future studies evaluating cerebral perfusion. The testing of multiple different contrast doses and bolus rates were beyond the scope of this study. Additional studies with a large population of normal dogs may help to determine if any alterations to the recommended dose and rate could be implemented. Gadolinium is a considered a safe contrast medium; doubling 
the dose is common in pediatric human medicine (1). Thus, the current recommended dose for small dogs should have minimal ill effect to the patient and provide consistent diagnostic DSCMRI perfusion maps.

It is the authors' belief that the addition of perfusion maps to conventional MRI images in canine patients will optimize the diagnostic accuracy of intracranial lesions and aid in treatment and prognosis as it has in human medicine. This is the study in veterinary medicine to describe a protocol for the use of DSCMRI in canine patients. This study used a 1.5-T MRI and a power injector to obtain repeatable, uniform perfusion maps. This study establishes a protocol for canine 1.5-T DSC-MRI imaging that is different in large and small dogs. These protocols may be used for future applications to evaluate hemodynamic disturbances in canine intracranial pathology.

\section{REFERENCES}

1. Huisman TA, Sorensen AG. Perfusion-weighted magnetic resonance imaging of the brain: techniques and application in children. Eur Radiol (2004) 14(1):59-72. doi:10.1007/s00330-003-1972-y

2. Essig M, Nguyen TB, Shiroishi MS, Saake M, Provenzale JM, Enterline DS, et al. Perfusion MRI: the five most frequently asked clinical questions. AJR Am J Roentgenol (2013) 201(3):W495-510. doi:10.2214/AJR.12.9544

3. Keston P, Murray AD, Jackson A. Cerebral perfusion imaging using contrast-enhanced MRI. Clin Radiol (2003) 58(7):505-13. doi:10.1016/ S0009-9260(03)00130-2

4. Rosen BR, Belliveau JW, Aronen HJ, Kennedy D, Buchbinder BR, Fischman A, et al. Susceptibility contrast imaging of cerebral blood volume: human experience. Magn Reson Med (1991) 22(2):293-9; discussion 300-3. doi:10.1002/ mrm.1910220227

5. Donahue KM, Krouwer HG, Rand SD, Pathak AP, Marszalkowski CS, Censky SC, et al. Utility of simultaneously acquired gradient-echo and spin-echo cerebral blood volume and morphology maps in brain tumor patients. Magn Reson Med (2000) 43(6):845-53. doi:10.1002/1522-2594(200006)43:6<845::AID-MRM10>3.0.CO;2-J

6. Quarles CC, Ward BD, Schmainda KM. Improving the reliability of obtaining tumor hemodynamic parameters in the presence of contrast agent extravasation. Magn Reson Med (2005) 53(6):1307-16. doi:10.1002/mrm.20497

7. Gossmann A, Helbich TH, Kuriyama N, Ostrowitzki S, Roberts TP, Shames $\mathrm{DM}$, et al. Dynamic contrast-enhanced magnetic resonance imaging as a surrogate marker of tumor response to anti-angiogenic therapy in a xenograft model of glioblastoma multiforme. J Magn Reson Imaging (2002) 15(3):233-40. doi:10.1002/jmri.10072

8. Zhao Q, Lee S, Kent M, Schatzberg S, Platt S. Dynamic contrast-enhanced magnetic resonance imaging of canine brain tumors. Vet Radiol Ultrasound (2010) 51(2):122-9. doi:10.1111/j.1740-8261.2009.01635.x

9. Keir SL, Wardlaw JM. Systematic review of diffusion and perfusion imaging in acute ischemic stroke. Stroke (2000) 31(11):2723-31. doi:10.1161/01. STR.31.11.2723

10. Hoggard N, Wilkinson ID, Griffiths PD. The imaging of ischaemic stroke. Clin Radiol (2001) 56(3):171-83. doi:10.1053/crad.2000.0619

11. Knopp MV, Essig M, Hawighorst H, Wenz F, Brix G, Schad LR, et al. Functional neuroimaging in the assessment of CNS neoplasms. Eur Radiol (1997) 7(Suppl 5):209-15. doi:10.1007/PL00006894

12. Law M, Cha S, Knopp EA, Johnson G, Arnett J, Litt AW. High-grade gliomas and solitary metastases: differentiation by using perfusion and proton spectroscopic MR imaging. Radiology (2002) 222(3):715-21. doi:10.1148/ radiol.2223010558

13. Cha S. Update on brain tumor imaging: from anatomy to physiology. Am J Neuroradiol (2006) 27(3):475-87.

14. Bozzao A, Floris R, Baviera ME, Apruzzese A, Simonetti G. Diffusion and perfusion MR imaging in cases of Alzheimer's disease: correlations with cortical atrophy and lesion load. AJNR Am J Neuroradiol (2001) 22(6):1030-6.

\section{AUTHOR CONTRIBUTIONS}

KS: principal investigator and primary author of the manuscript. As PI, KS was responsible for research development and adjustment of the methods, grantsmanship, and authoring of the manuscript. This research was done as part of KS's radiology residency. AP and EB: both faculty resident and research mentors responsible for editing and approving methodology prior to initiation, editing, and co-PI on grants and IACUC, as well as internal editing of the manuscript.

\section{FUNDING}

This study was supported by Michigan State University College of Veterinary Medicine Endowed Research Grant.

15. Lorenz IH, Kolbitsch C, Hormann C, Luger TJ, Schocke M, Eisner W, et al. The influence of nitrous oxide and remifentanil on cerebral hemodynamics in conscious human volunteers. Neuroimage (2002) 17(2):1056-64. doi:10.1006/ nimg.2002.1228

16. O'Brien TJ, David EP, Kilpatrick CJ, Desmond P, Tress B. Contrast-enhanced perfusion and diffusion MRI accurately lateralize temporal lobe epilepsy: a pilot study. J Clin Neurosci (2007) 14(9):841-9. doi:10.1016/j.jocn.2006.07.003

17. Beaumont A, Fatouros P, Gennarelli T, Corwin F, Marmarou A. Bolus tracer delivery measured by MRI confirms edema without blood-brain barrier permeability in diffuse traumatic brain injury. Acta Neurochir Suppl (2006) 96:171-4. doi:10.1007/3-211-30714-1_38

18. Dagal A, Lam AM. Cerebral blood flow and the injured brain: how should we monitor and manipulate it? Curr Opin Anaesthesiol (2011) 24(2):131-7. doi:10.1097/ACO.0b013e3283445898

19. Law M, Yang S, Wang H, Babb JS, Johnson G, Cha S, et al. Glioma grading: sensitivity, specificity, and predictive values of perfusion MR imaging and proton MR spectroscopic imaging compared with conventional MR imaging. AJNR Am J Neuroradiol (2003) 24(10):1989-98.

20. Lev MH, Ozsunar Y, Henson JW, Rasheed AA, Barest GD, Harsh GRT, et al. Glial tumor grading and outcome prediction using dynamic spin-echo MR susceptibility mapping compared with conventional contrast-enhanced MR: confounding effect of elevated rCBV of oligodendrogliomas [corrected]. AJNR Am J Neuroradiol (2004) 25(2):214-21.

21. Law M, Oh S, Babb JS, Wang E, Inglese M, Zagzag D, et al. Low-grade gliomas: dynamic susceptibility-weighted contrast-enhanced perfusion MR imaging - prediction of patient clinical response. Radiology (2006) 238(2):658-67. doi:10.1148/radiol.2382042180

22. Law M, Young RJ, Babb JS, Peccerelli N, Chheang S, Gruber ML, et al. Gliomas: predicting time to progression or survival with cerebral blood volume measurements at dynamic susceptibility-weighted contrast-enhanced perfusion MR imaging. Radiology (2008) 247(2):490-8. doi:10.1148/radiol.2472070898

23. Server A, Kulle B, Gadmar OB, Josefsen R, Kumar T, Nakstad PH. Measurements of diagnostic examination performance using quantitative apparent diffusion coefficient and proton MR spectroscopic imaging in the preoperative evaluation of tumor grade in cerebral gliomas. Eur J Radiol (2011) 80(2):462-70. doi:10.1016/j.ejrad.2010.07.017

24. Hilario A, Ramos A, Perez-Nunez A, Salvador E, Millan JM, Lagares A, et al. The added value of apparent diffusion coefficient to cerebral blood volume in the preoperative grading of diffuse gliomas. AJNR Am J Neuroradiol (2012) 33(4):701-7. doi:10.3174/ajnr.A2846

25. Harris AD, Kosior JC, Ryder RC, Andersen LB, Hu WY, Hudon M, et al. MRI of ischemic stroke in canines: applications for monitoring intraarterial thrombolysis. J Magn Reson Imaging (2007) 26(6):1421-8. doi:10.1002/jmri. 21189

26. Liu R, Li X, Hu CL, Jiang L, Dai G, Wu GF, et al. The changes of brain water diffusion and blood flow on diffusion-weighted and perfusion-weighted imaging in a canine model of cardiac arrest. Resuscitation (2012) 83(5):645-51. doi:10.1016/j.resuscitation.2011.10.017 
27. Tidwell AS, Robertson ID. Magnetic resonance imaging of normal and abnormal brain perfusion. Vet Radiol Ultrasound (2011) 52(1 Suppl 1):S62-71. doi:10.1111/j.1740-8261.2010.01786.x

28. Dickinson PJ. Advances in diagnostic and treatment modalities for intracranial tumors. J Vet Intern Med (2014) 28(4):1165-85. doi:10.1111/jvim.12370

29. Chen JJ, Rosas HD, Salat DH. Age-associated reductions in cerebral blood flow are independent from regional atrophy. Neuroimage (2011) 55(2):468-78. doi:10.1016/j.neuroimage.2010.12.032

30. de Lange C, Brabrand K, Emblem KE, Bjornerud A, Loberg EM, Saugstad $\mathrm{OD}$, et al. Cerebral perfusion in perinatal hypoxia and resuscitation assessed by transcranial contrast-enhanced ultrasound and $3 \mathrm{~T}$ MRI in newborn pigs. Invest Radiol (2011) 46(11):686-96. doi:10.1097/RLI.0b013e3182266431

31. Lobel U, SedlacikJ, Reddick WE, Kocak M, Ji Q, Broniscer A, et al. Quantitative diffusion-weighted and dynamic susceptibility-weighted contrast-enhanced perfusion MR imaging analysis of T2 hypointense lesion components in pediatric diffuse intrinsic pontine glioma. AJNR Am J Neuroradiol (2011) 32(2):315-22. doi:10.3174/ajnr.A2277

32. Cha S. Perfusion MR imaging of brain tumors. Top Magn Reson Imaging (2004) 15(5):279-89. doi:10.1097/00002142-200410000-00002

Conflict of Interest Statement: The authors declare that the research was conducted in the absence of any commercial or financial relationships that could be construed as a potential conflict of interest.

Copyright (c) 2017 Stadler, Pease and Ballegeer. This is an open-access article distributed under the terms of the Creative Commons Attribution License (CC BY). The use, distribution or reproduction in other forums is permitted, provided the original author(s) or licensor are credited and that the original publication in this journal is cited, in accordance with accepted academic practice. No use, distribution or reproduction is permitted which does not comply with these terms. 